\title{
Spanwise boundary layer modulations using finite discrete suction for transition delay
}

\author{
Sohrab S. Sattarzadeh ${ }^{1} \cdot$ Jens H. M. Fransson ${ }^{1}$ (])
}

Received: 15 September 2016 / Revised: 3 January 2017 / Accepted: 7 January 2017 / Published online: 7 February 2017

(C) The Author(s) 2017. This article is published with open access at Springerlink.com

\begin{abstract}
Discrete suction is deployed in a flat plate boundary layer to create spanwise mean velocity gradients (SVG) with the goal of delaying the onset of laminar-toturbulent transition. It is shown that finite boundary layer suction through a set of holes in a spanwise oriented array in the flat plate is successful in setting up steady and robust streamwise streaks in the boundary layer. Today, the SVG method for transition control is known to attenuate the growth of Tollmien-Schlichting (TS) waves and delay the transition to turbulence. In this investigation, low-amplitude forced TS waves are attenuated with the implication of extending the laminar flow by at least $120 \%$ for a discrete suction of $0.8 \%$ of the free-stream velocity. The control technique is also tested successfully for natural transition, with a resulting transition delay of $30 \%$.
\end{abstract}

\section{Introduction}

The spanwise mean velocity gradient (SVG) method is proven to serve as a laminar flow control technique for attenuating the boundary layer disturbances and delaying the transition to turbulence in flat plate boundary layer. Extending the laminar boundary layer in the streamwise direction, by means of flow control, is of great interest (Green 2008), since the local skin-friction coefficient is typically an order of magnitude lower in laminar-compared to turbulent boundary layers for relevant Reynolds numbers. This, in addition to the principal role of skin-friction

Jens H. M. Fransson

jensf@mech.kth.se

1 Linné Flow Centre, KTH Royal Institute of Technology, 10044 Stockholm, Sweden drag in the total drag budget for aerodynamically smooth bodies (Joslin 1998), explains the vast interest in developing new technologies to reduce skin-friction drag.

The application of wall-suction systems has been known, since long, to stabilize boundary layers (see, e.g., Gad-el-Hak 2000). Laminar flow control aimed at drag reduction using suction through spanwise slots, and porous panels or discrete holes have been applied in the past (see, e.g., Pfenninger and Groth 1961; Reynolds and Saric 1986; MacManus and Eaton 2000; Roberts et al. 2001). Wall suction bring high momentum fluid, in the upper shear layer, towards the wall and creates a fuller mean velocity profile, i.e., a profile with lower shape factor, compared to a Blasius boundary layer. Two-dimensional theoretical modalstability analysis predicts a progressively more stable boundary layer as the shape factor decreases. When considering steady boundary layer suction over a large area, the boundary layer will asymptote to the specific shape of an exponential profile with a shape factor of 2 . This state is the so-called asymptotic suction state, and its mean velocity profile can readily be derived in an explicit-analytic form (see, e.g., Schlichting 1979). Once the asymptotic suction boundary layer (ASBL) is reached the shape factor of 2 will not be altered even if the suction velocity at the wall is increased. However, increased suction results in a thinner boundary layer with the implication of a higher skin-friction drag. The critical Reynolds number for the ASBL is $R e_{\text {cr }}=5.4 \times 10^{4}$ (see, e.g., Drazin and Reid 1981; Fransson and Alfredsson 2003) and in the asymptotic limit the suction coefficient $\left(C_{q}\right)$ is equal to the inverse of the Reynolds number. This means that $C_{q}=1.85 \times 10^{-5}$ ensures that the boundary layer remains stable for all frequencies at the lowest possible skin-friction drag, i.e., at the lowest suction rate. Worth mentioning in this context is that the entry length in reaching the asymptotic state is not 
considered. During the spatial development of the boundary layer, towards the asymptotic state, the boundary layer is more vulnerable to external perturbations, and hence, a higher suction coefficient may be needed to actually keep the boundary layer laminar. In addition, it should be noted that the theoretical value is not meaningful when the free stream is subjected to free-stream turbulence. Fransson and Alfredsson (2003) and Yoshioka et al. (2004) report disturbance energy decay inside the boundary layer at $C_{q}$ values of $2.9 \times 10^{-3}$ and $4.0 \times 10^{-3}$ for turbulence intensity values in the range of $1.4-1.6$ and $2.0-2.3 \%$, respectively. That is, two orders of magnitude larger suction rate are required to attenuate boundary layer disturbances with free-stream turbulence compared to undisturbed free stream. Note that, to make comparisons with discrete suction holes (as used in the present study), one has to consider the volume flow rate over a downstream region, where laminar flow can be certified. Gregory (1961) reviewed various types of suction surfaces, among them suction through discrete perforations. A modern manufacturing process of such perforated surfaces is to use laser drilling through metal sheets. A porosity of $0.5 \%$ is typically enough to make the flow, over the surface, experience a uniform suction (see, e.g., Trip and Fransson 2014, where the laser drilled holes had an averaged diameter of $60 \mu \mathrm{m})$. This gives a volume-suction coefficient of $C_{q}^{*}=C_{q} \cdot\left(5 \times 10^{-3}\right)=9.26 \times 10^{-8}$ for later comparison.

The SVG method is based on the formation of streamwise elongated high- and low-speed regions in the boundary layer which are often denoted streamwise streaks. The spanwise gradients that are generated in the mean flow in the presence of the high- and low-speed regions alter the evolution of Tollmien-Schlichting (TS) waves in the boundary layer. TS waves are two-dimensional (2D) sinusoidal disturbance waves which are the main source of boundary layer transition to turbulence in low background disturbance environments (Tollmien 1929; Schlichting 1933; Schubauer and Skramstad 1947).

These waves are the most unstable eigenmodes to the linear stability problem (Squire 1933). The physical mechanism involved in the attenuation of TS waves in streaky boundary layers may be explained using the governing equations describing the physical processes of the flow. With an additional spanwise mean velocity gradient, due to the steady streaks, there will be an extra production term in the perturbation kinetic energy equation associated with the streamwise-spanwise covariance acting on the additional spanwise mean velocity gradient. This additional term together with the viscous dissipation counteracts the wallnormal production term that is responsible for the amplitude growth of TS waves in 2D boundary layers (Cossu and Brandt 2004).

The attenuation of low-amplitude TS wave disturbances due to the presence of spanwise mean velocity variations has been reported both experimentally (Kachanov and Tararykin 1987; Westin et al. 1994; Boiko et al. 1994; Fransson et al. 2005a; Sattarzadeh and Fransson 2015) and numerically (Cossu and Brandt 2002, 2004; Siconolfi et al. 2015b). It is also reported in both experimental (Fransson et al. 2006; Shahinfar et al. 2012, 2013, 2014; Sattarzadeh et al. 2014; Sattarzadeh and Fransson 2014; Downs and Fransson 2014) and numerical (Schlatter et al. 2010; Siconolfi et al. 2015a) investigations that the attenuation effect can be extended to high-amplitude non-linear TS waves which leads to delay of laminar-to-turbulence transition in streaky boundary layers.

The amplitude of the streamwise streaks determines how effective the TS waves are attenuated for a given spanwise wavelength. A crucial condition, however, for successfully being able to apply the SVG method experimentally is the stability of the generated streamwise streaks. The spanwise modulations in the boundary layer undergo algebraic disturbance growth, which is described by the lift-up mechanism (Landahl 1980). This growth is followed by an exponential decay due to the viscous effects in the boundary layer (Luchini 1996, 2000). However, if the disturbance amplitude reaches a critical value, the streaks become susceptible to a secondary instability (Andersson et al. 1999) that forces them to break down to turbulence. Various techniques have been used to experimentally generate steady streamwise streaks in the boundary layer. Tani and Komoda (1962) employed small wings located outside of the boundary layer, and Kachanov and Tararykin (1987) applied spanwise periodic blowing and suction through wall slots. The employment of a spanwise periodic array of roughness elements of different shapes has also been a successful method to generate stable streaks (Bakchinov et al. 1995; White 2002; Fransson et al. 2004, 2005a, 2006; White et al. 2005). A breakthrough for generating high-amplitude stable streaks in the boundary layer was the method proposed by Fransson and Talamelli (2012), where the conventional roughness elements were replaced by miniature vortex generators (MVGs).

Other means to generate spanwise modulations to trigger the SVG method, without the presence of wall-mounted boundary-layer modulators, have also been tested successfully. Downs and Fransson (2014) created spanwise velocity gradients by directly shifting the wall location periodically in the spanwise direction using streamwise elongated humps. In this way, the no-slip boundary condition guarantees the desired modulation, which led to transition delay. Free-stream vortices is another means that have been used successfully for transition delay (Siconolfi et al. 2015b). These vortices penetrate the boundary layer further downstream and creates the modulation from the free stream. Artificially generated pair of oblique waves (POW) have also proven to be effective in attenuating the growth of TS 
waves (Sattarzadeh and Fransson 2016). The POW interacts non-linearly and can create steady and stable streamwise streaks under controlled conditions which leads to an extension of the laminar flow region of up to $230 \%$.

Even though all the investigated methods were successful in generating steady streaks in the boundary layer, most of them share a drawback regarding the employment of fixed physical devices that may in critical conditions accelerate the boundary layer transition to turbulence. In addition, there are applications, where protrusive devices are not suitable or not even allowed. Therefore, investigating new methods for generating streamwise streaks without the need of physical devices would be of interest, where the control can instantaneously be turn on and off (just as in the POW method). In the present study, we investigate the possibility of generating high-amplitude stable streaks by applying finite discrete suction in the boundary layer through a set of spanwise-oriented array of holes. A similar method has previously been used to investigate the stability of streamwise streaks in plane Poiseuille flow by Elofsson et al. (1999). While the flow physics of discrete boundary layer suction was studied intensively by MacManus and Eaton (2000), here, the focus of the present investigation is on the stabilizing effect of the streaks generated by the discrete suction on TS waves in flat plate boundary layers in both linear and non-linear regimes (i.e., for both small and large TS-wave forcing amplitudes).

\section{Experimental setup}

The experiments were conducted in the Minimum-Turbulence-Level (MTL) wind tunnel at KTH-Royal Institute of Technology. The MTL is a closed circuit wind tunnel, where the flow is provided by an axial fan powered by an $85 \mathrm{~kW}$ DC electric motor. The test section is $7 \mathrm{~m}$ long with a cross section of $0.8 \mathrm{~m}$ in height and $1.2 \mathrm{~m}$ in width. The turbulence level of MTL is less than $0.025 \%$ in the streamwise direction at a nominal speed of $25 \mathrm{~m} \mathrm{~s}^{-1}$. The high flow quality of the tunnel in terms of low background disturbance level and high flow homogeneity provides suitable conditions for stability experiments (see Lindgren and Johansson 2002, for further details). The circulating air is maintained at constant temperature, with a tolerance of $\pm 0.05^{\circ} \mathrm{C}$ for velocities above $6 \mathrm{~m} \mathrm{~s}^{-1}$, by means of a heat exchanger installed downstream of the fan.

The measurements were performed in a flat plate boundary layer. A sketch of the flat plate mounted horizontally in the wind tunnel test section is shown in Fig. 1a, where the coordinate system $(x, y, z)$ is introduced. The streamwise velocity component is denoted by $U$ with the small letter corresponding to the disturbance velocity. The external (a)

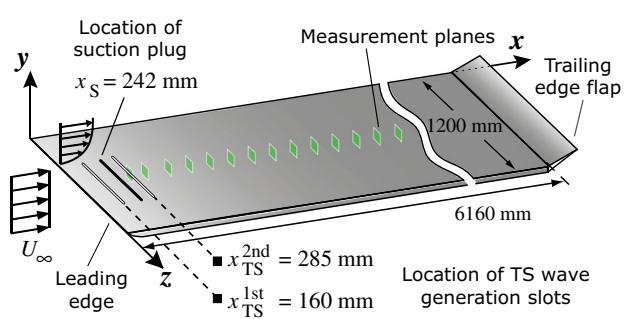

(b)
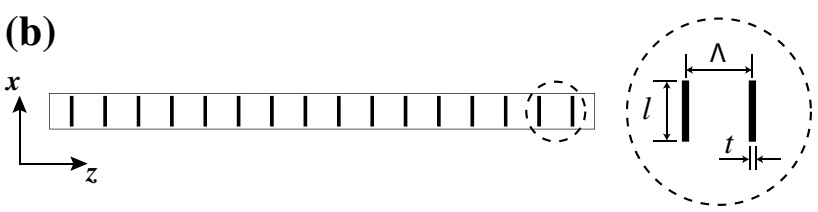

Fig. 1 a Schematic of the flat plate mounted horizontally in the wind tunnel test section. Locations of TS wave generation slots are depicted in the figure as $x_{\mathrm{TS}}^{1 \text { st }}$ and $x_{\mathrm{TS}}^{2 \text { nd }}$. The downstream edges of the suction holes are located at $x_{\mathrm{S}}$. The cross-sectional measurement planes are also depicted. b Schematic of the suction plug from the top view. The inset shows a close view of two suction holes with the geometrical parameters $l=10 \mathrm{~mm}$ and $t=1 \mathrm{~mm}$. The spanwise wavelength of the suction holes in the array is $\Lambda=12 \mathrm{~mm}$

pressure gradient along the plate was set by the adjustable ceiling in the test section.

Single-probe hot-wire anemometry measurements with temporal and spatial resolutions of $5 \mathrm{kHz}$ and $0.6 \mathrm{~mm}$, respectively, were carried out in cross-sectional $y z$ planes $(21 \times 12$ measurement points) in the boundary layer. Single hot-wire probes, employed for measuring the streamwise component of the velocity, were manufactured in-house with Wollaston Platinum wire with a diameter of $2.54 \mu \mathrm{m}$ and length of $0.6 \mathrm{~mm}$. A DANTEC Dynamics ${ }^{\mathrm{TM}}$ StreamLine 90N10 anemometer system was used, where the voltage signals were acquired by a National Instruments ${ }^{\mathrm{TM}}$ acquisition board (NI PCI-6259, 16-Bit). The calibration of the hot-wire probes was performed in-situ versus a Prandtl tube connected to a differential pressure manometer (Furness FC0510). A modified King's law (Johansson and Alfredsson 1982) was used as the calibration function to improve measurements in low velocities close to the plate. The hot-wire probe was traversed by a computer-controlled traversing system with three degrees of freedom.

\subsection{Reference Blasius boundary layer}

The external pressure gradient along the flat plate was close to nullified for a free-stream velocity of $U_{\infty}=6 \mathrm{~m} \mathrm{~s}^{-1}$, by adjusting the ceiling of the test section, to obtain a Blasius boundary layer over the flat plate. The streamwise distribution of the pressure coefficient $C_{\mathrm{p}}=1-\left(U_{e} / U_{\infty}\right)^{2}$ is shown in Fig. 2a, where it is depicted that disregarding a minimal suction peak on the upper side of the plate close to the leading edge, the pressure coefficient variation lies 

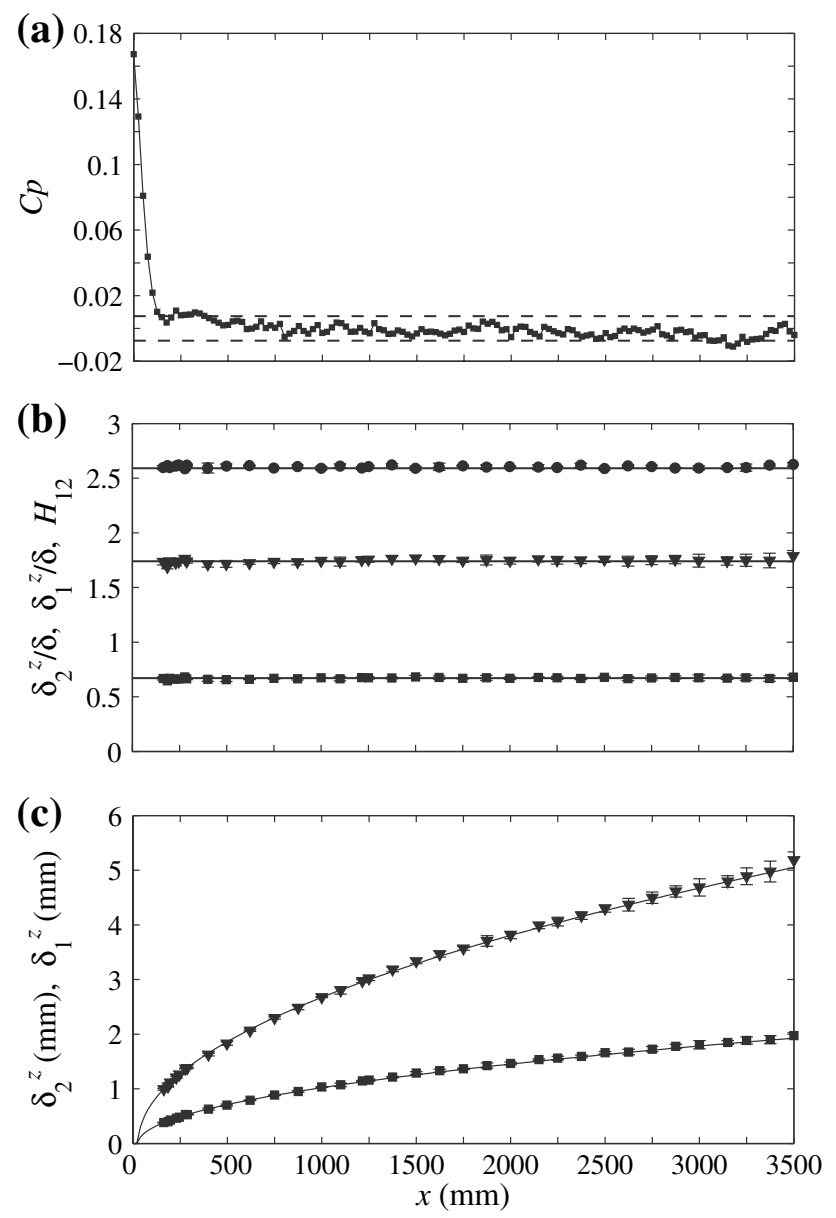

Fig. 2 Mean flow characteristics at $U_{\infty}=6 \mathrm{~m} \mathrm{~s}^{-1}$. a Pressure coefficient distribution along the streamwise direction. Dashed lines mark the pressure coefficient variation of $\Delta C_{\mathrm{p}}=0.15$. b Spanwise averaged boundary layer parameters $H_{12}^{z}$ (filled circle), $\delta_{1}^{z}$ (filled down triangle) and $\delta_{2}^{z}$ (filled square), where the displacement and momentum thicknesses are normalized by the boundary layer scale $\delta$.Error bars represent the standard deviation of the values measured in three spanwise locations. Solid lines mark the theoretical Blasius boundary layer parameters $H_{12}=2.592, \delta_{1} / \delta=1.721$ and $\delta_{2} / \delta=0.664$. c Unscaled values of $\delta_{1}^{z}$ (filled down triangle) and $\delta_{2}^{z}$ (filled square). The solid lines mark theoretical Blasius boundary layer values adjusted for a virtual origin at $x=27 \mathrm{~mm}$ within \pm 0.075 down to $x=3500 \mathrm{~mm}$. The external pressure gradient influences the evolution of the integral boundary layer parameters such as displacement thickness, $\delta_{1}$, momentum thickness, $\delta_{2}$, and hence the shape factor $H_{12}\left(=\delta_{1} / \delta_{2}\right)$. For a self-similar boundary layer the displacement thickness and momentum thickness would exhibit a constant value in the streamwise direction when normalized by the boundary layer scale $\delta\left(=\sqrt{v x / U_{\infty}}\right)$. Here $v$ is the kinematic viscosity. The spanwise averaged boundary layer parameters $\delta_{1}^{z}$ (displacement thickness), $\delta_{2}^{z}$ (momentum thickness) and $H_{12}^{z}$ (shape factor) for the reference boundary layer are shown in Fig. 2b, where the error bars represent the standard deviation of the values measured in three spanwise locations: $z=[-48,0,48] \mathrm{mm}$. The theoretical Blasius boundary layer parameters $H_{12}=2.592$, $\delta_{1} / \delta=1.721$ and $\delta_{2} / \delta=0.664$ are displayed as solid lines. Dimensional values of $\delta_{1}^{z}$ and $\delta_{2}^{z}$ are shown in Fig. 2c with theoretical Blasius values adjusted for a virtual origin at $x=27 \mathrm{~mm}$.

\subsection{Generation of the streamwise streaks}

The streamwise streaks were generated by means of spanwise periodic finite suction through an array of 16 holes in a spanwise-oriented plug flush mounted in the flat plate. A schematic of the suction plug is depicted in Fig. 1b. A similar method has been previously used for investigating the stability of streamwise streaks in plane Poiseuille flow by Elofsson et al. (1999). The suction holes had the dimensions $1 \mathrm{~mm} \times 10 \mathrm{~mm}$ (width $\times$ length) and their downstream edge was located at $x_{\mathrm{S}}=242 \mathrm{~mm}$. The spanwise wavelength of the suction holes in the array was $\Lambda=12 \mathrm{~mm}$ which renders $\beta_{0}=0.40$ for $U_{\infty}=6 \mathrm{~m} \mathrm{~s}^{-1}$. Here $\beta_{0}$ is the non-dimensional spanwise wavenumber defined as $\beta_{0}=2 \pi \delta_{0} / \Lambda$, where $\delta_{0}$ is the boundary layer scale at $x_{\mathrm{S}}$. The holes were connected to a low-pressure tank which in turn was connected to the inlet of a centrifugal fan. The suction rates at the holes were adjusted by clamps mounted around the tubes and were individually regulated to give a purely sinusoidal spanwise profile for each case. Figure 3 shows the spanwise distribution of the normalized mean streamwise velocity at $x-x_{\mathrm{S}}=78 \mathrm{~mm}$ and wall-normal location of
Fig. 3 Spanwise distribution of the normalized mean streamwise velocity (filled circle) at the downstream and wallnormal location of maximum modulation for the medium suction rate with $v_{0} / U_{\infty}=2.3 \%$. The (open circle) symbols correspond to the reference Blasius boundary layer mean flow at the same location

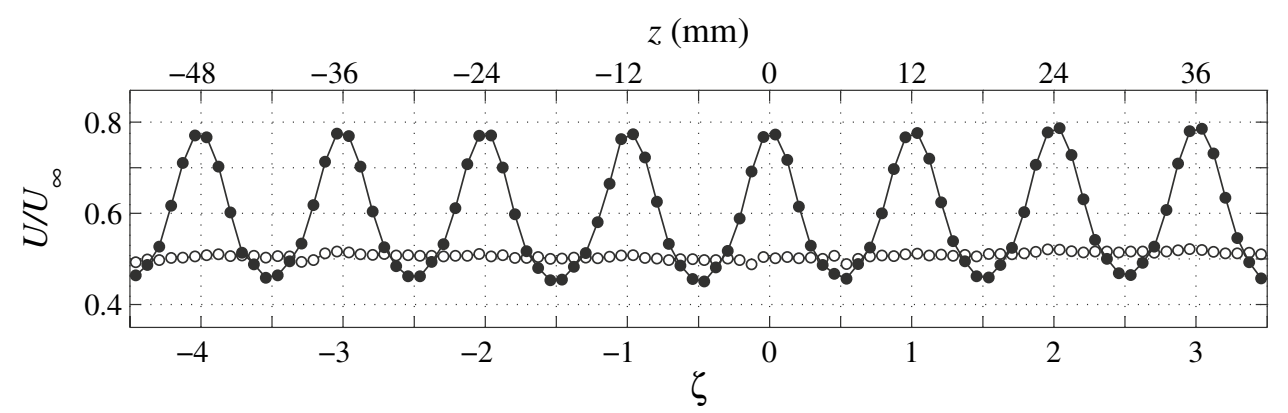


maximum modulation, where $\zeta=z / \Lambda$ and the origin of the spanwise coordinate $\zeta=0$ is located at the center of a suction hole. The total suction rate for each investigated case was measured at the outlet of the centrifugal fan by a R-2-15-C glass-float Sho-Rate purgemeter with a maximum range of $65.7 \mathrm{~cm}^{3} \mathrm{~s}^{-1}$ for air at $20^{\circ} \mathrm{C}$ and atmospheric pressure. Three suction rates of $v_{0} / U_{\infty}=0.8,2.3$, and 3.9\% were investigated at $U_{\infty}=6 \mathrm{~m} \mathrm{~s}^{-1}$. Here $v_{0}=\left|V_{0}\right|$ with $V_{0}<0$ being the suction velocity at each hole.

\subsection{Generation of Tollmien-Schlichting waves}

The artificially forced TS waves were introduced by means of blowing and suction through two sets of 36 tubes plugged into two spanwise-oriented disturbance slots in the flat plate at $x_{1 \mathrm{st}}^{\text {dist. }}=160 \mathrm{~mm}$ and $x_{2 \text { did }}^{\text {dist }}=285 \mathrm{~mm}$, as depicted in Fig. 1 (see Gaponenko and Kachanov 1994, where a similar slot was used). Two measurement configurations were investigated consisting of the TS wave generation upstream $(\mathrm{C} 1)$ and downstream $(\mathrm{C} 2)$ of the discrete suction plug. The disturbance slots were connected to a loudspeaker driven by sinusoidal voltage signals. The frequency, phase, and amplitude of the signals were controlled by a computer. The artificially generated boundary layer disturbances hence rendered uniform sinusoidal waves in the spanwise direction. For the excited disturbances, we use the non-dimensional frequency $F=\left(2 \pi f_{0} v / U_{\infty}^{2}\right) \times 10^{6}$ throughout this paper, where $f_{0}$ is the forcing frequency in $\mathrm{Hz}$.

\section{The spanwise modulated boundary layer}

The discrete suction on the surface of the flat plate introduces a spanwise periodic steady disturbance in the mean flow which undergoes a non-modal transient growth in the shear layer. The suction $v_{0}$ applied at the location of each discrete hole pulls the boundary layer toward the wall which forms circulation regions and generates sets of counter-rotating streamwise vortices with common down flow at the spanwise location of the suction holes. The counter-rotating vortices modulate the boundary layer in the spanwise direction by pushing the high momentum flow toward the wall downstream of the suction holes and the low momentum flow away from the wall in the spanwise region between the suction holes. Consequently, streamwise elongated streaks of high and low speed are generated in the boundary layer with the same spanwise wavelength as the holes in the suction plug through the lift-up mechanism. The spanwise modulated mean flow downstream of one suction hole is shown in Fig. 4 for three suction rates $v_{0} / U_{\infty}=0.8,2.3$ and $3.9 \%$ at $x-x_{\mathrm{S}}=3 \mathrm{~mm}$. Here, the wall-normal direction is normalized with the boundary layer scale as $\eta=y / \delta$. It is shown that the initial modulation of the mean flow, at a short distance downstream of the suction plug, is increased for higher suction rates due to higher induced circulation in the cross-sectional plane.

As a result of the streamwise streaks, the integral boundary layer parameter values $\delta_{1}, \delta_{2}$, and $H_{12}$ are altered in the spanwise modulated boundary layer compared to the ones for the reference Blasius boundary layer, as shown in Fig. 2b. Figure 5a-c displays $\delta_{1}, \delta_{2}$, and $H_{12}$, respectively, in the $x z$-plane for one spanwise wavelength downstream of one suction hole and for the highest suction rate $v_{0} / U_{\infty}=3.9 \%$. It is shown that the boundary layer modulations are symmetric in the spanwise direction about $\zeta=0$. The spanwise averaged parameters $\delta_{1}^{z} / \delta, \delta_{2}^{z} / \delta$, and $H_{12}^{z}$ are displayed in Fig. 5d, where the theoretical Blasius boundary layer parameters are depicted by dashed dotted lines. Noteworthy is that in the presence of discrete and spanwise

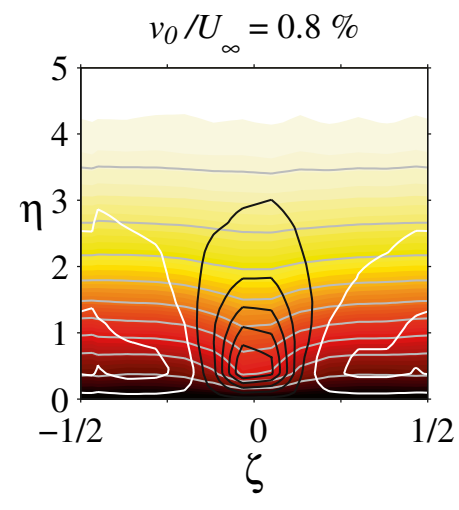

Fig. 4 Cross-sectional contour plots of the streamwise mean flow for three suction rates $v_{0} / U_{\infty}=0.8,2.3$ and $3.9 \%$ from left to right, respectively, at $x-x_{\mathrm{S}}=3 \mathrm{~mm}$. The gray lines mark the contours of constant velocity (0.1:0.1:0.9), and the black and white contour lines display the regions of velocity excess $(0.01: 0.03: 0.4)$ and velocity deficit (-0.01:-0.03:-0.3), respectively, with respect to the spanwise averaged mean flow 
(a)

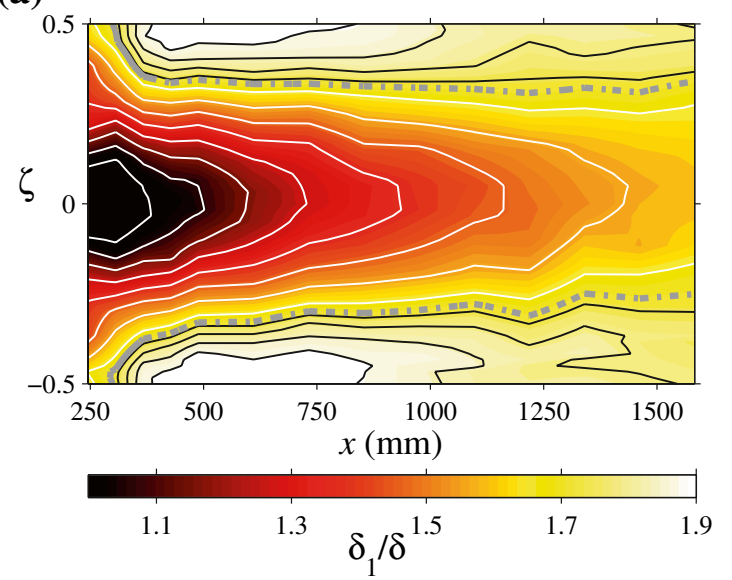

(c)

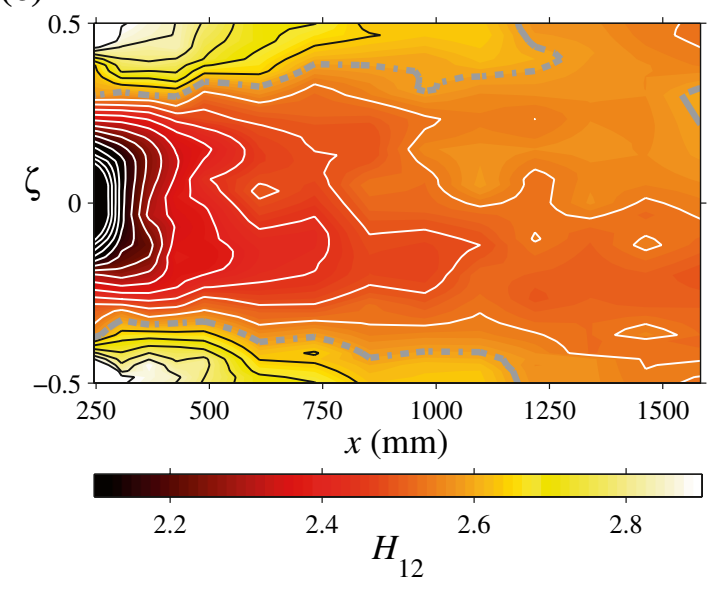

(b)

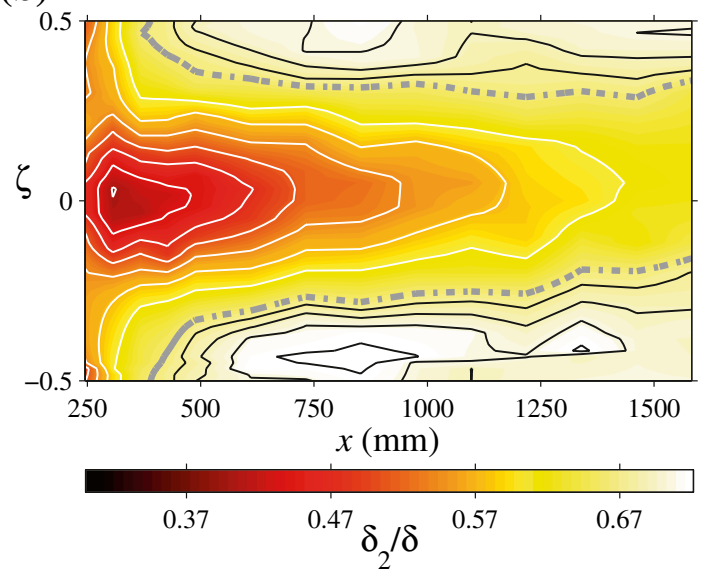

(d)

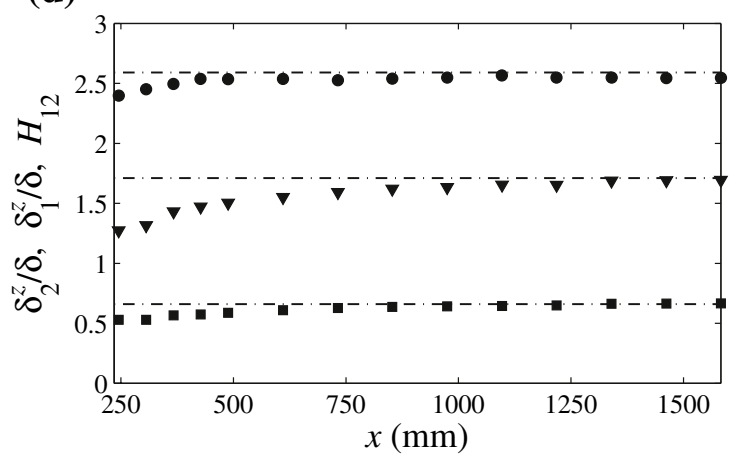

Fig. 5 Boundary layer parameters for the high suction rate with $v_{0} / U_{\infty}=3.9 \%$. a-c display the contours of $\delta_{1} / \delta, \delta_{2} / \delta$ and $H_{12}$, respectively. The gray dashed lines mark the area corresponding to the value of theoretical Blasius boundary layer parameters and the

distributed suction, the integral parameters deviate considerably from the Blasius values as far downstream as $x \approx 750 \mathrm{~mm}$ whereafter the deviation diminishes.

As depicted in Fig. 5a-c the low-speed regions, i.e., those displayed by the white colour, are generated in the spanwise region between the suction holes $(\zeta= \pm 0.5)$ from the initial stage. This is in contrast with the streaky boundary layers generated by means of physical devices mounted on the flat plate, e.g., roughness elements and MVGs, where the low-speed regions are initially generated in the wake of these devices which render a complex modulation of the boundary layer with a wide range of spanwise modes in the near wakes. This is further illustrated in Fig. 6a, where the mean flow modulation with respect to the Blasius boundary layer $\left(U_{B}\right.$ ) is displayed as $U^{*}=\left(U-U_{\mathrm{B}}\right) / U_{\mathrm{B}}$ in the $x z$-plane downstream of four suction holes. Here, the mean flow is measured at the wall-normal location of maximum spanwise modulation for each downstream location. The amplitude of the

white and black solid lines mark the contours of excess and deficit from it. d The spanwise averaged parameters; $H_{12}^{z}$ (filled circle), $\delta_{1}^{z}$ (filled down triangle) and $\delta_{2}^{z}$ (filled square). The dashed-dotted lines mark the theoretical Blasius boundary layer parameters

corresponding spanwise Fourier modes $A\left(\lambda_{i}\right)$ is displayed in Fig. $6 \mathrm{~b}$ where $\lambda_{i}=\Lambda / i$ with $i=1,2,3$ is the spanwise wavelength of the steady disturbances excited in the mean flow. The $\lambda_{0}$-mode represents the spanwise-invariant modulations compared to the reference Blasius boundary layer. It is shown that fundamental mode $\lambda_{1}$, with the same wavelength as the suction holes, has the highest amplitude and is the dominating mode with its first two harmonics $\lambda_{2}$ and $\lambda_{3}$ decaying rapidly.

To investigate the evolution of the streamwise streaks, an integral measure of the boundary layer modulation over the entire cross-sectional $y z$-plane is utilized, similar to Shahinfar et al. (2013), as

$A_{\mathrm{ST}}^{\mathrm{int}}(x)=\int_{-1 / 2}^{+1 / 2} \int_{0}^{\eta^{*}} \frac{\left|U(x, \eta, \zeta)-U^{z}(x, \eta)\right|}{U_{\infty}} \mathrm{d} \eta \mathrm{d} \zeta$. 
Fig. 6 Boundary layer measurements at the wall-normal location of maximum spanwise modulation for the medium suction rate with $v_{0} / U_{\infty}=2.3 \%$. a Contour plot of mean flow modulation $U^{*}=\left(U-U_{\mathrm{B}}\right) / U_{\mathrm{B}}$ in the $x z$-plane downstream of four suction holes. b Normalized amplitudes of the spanwise Fourier modes of mean flow $A\left(\lambda_{i}\right) / U_{\infty}$

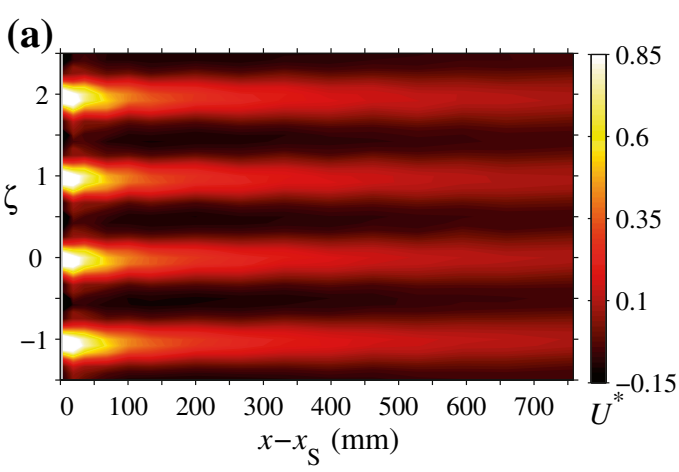

Here the truncation value for wall-normal integration is set to $\eta^{*}=9$, to capture most of the disturbance amplitude in a similar integral measure for the TS waves (defined in the next section), where the amplitude is significant even outside the boundary layer. Recall that the boundary layer thickness of the reference Blasius boundary layer is at $\eta \approx 5$. The streamwise evolution of the streak amplitude $A_{\mathrm{ST}}^{\text {int }}$ is shown in Fig. 7a for three suction rates $v_{0} / U_{\infty}=0.8$, 2.3 and $3.9 \%$. It is observed that the streaks initially undergo an algebraic amplitude growth and reach their maxima $A_{\mathrm{ST}}^{\text {int, max }}$ at some downstream location, whereafter the streak amplitude decays exponentially as a consequence of viscous dissipation. Higher $A_{\mathrm{ST}}^{\mathrm{int} \text { max }}$ are obtained for higher suction rates as shown in Fig. 7b. The streamwise location of the maximum streak amplitude is also pushed downstream by increasing the suction rate, since stronger streamwise vortices are generated which can modulate the mean flow further downstream prior to the location where the viscous effect sets in. The inset in Fig. 7a shows a magnified display of the streak amplitude evolution for $v_{0} / U_{\infty}=2.3 \%$ with two extra points measured upstream of the suction array. It is observed that the applied suction also (b)

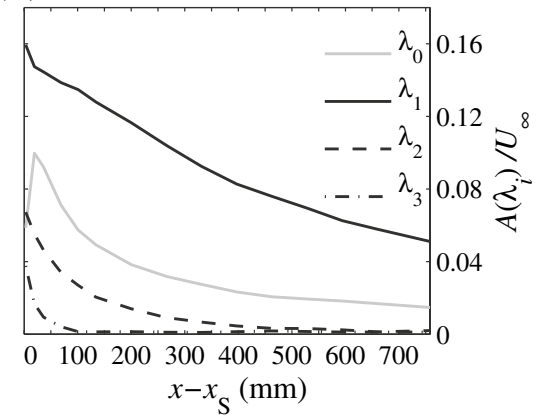

weakly modulates the mean flow field upstream of the suction plug.

\section{Attenuation of linear Tollmien-Schlichting waves}

In this section, we investigate the amplitude evolution of the linear TS waves in the streaky boundary layer generated by the discrete suction and compare it to the corresponding amplitude evolution in the reference Blasius boundary layer. The linear regime of the TS waves is corresponding to low-amplitude disturbances. To avoid non-linearities the local maximum amplitude of the TS waves at the $x$ location corresponding to branch II of the neutral stability curve is here set to $0.7 \%$ of $U_{\infty}$ for each investigated reference case. This criterion ensures that the non-linear effects, i.e., the harmonics of the forced TS wave frequency, are not triggered in the boundary layer.

The amplification curve of the TS waves is altered in the streaky boundary layer compared to the reference $2 \mathrm{D}$ boundary layer. Therefore, to investigate the disturbance

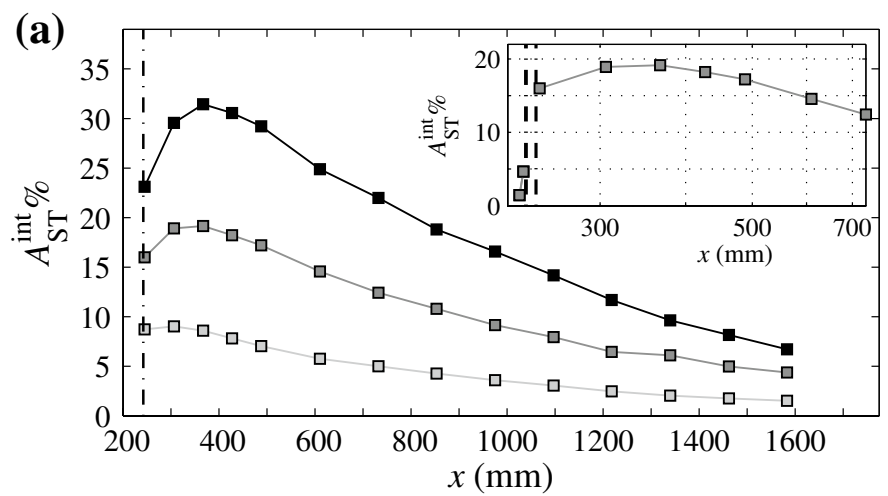

(b)

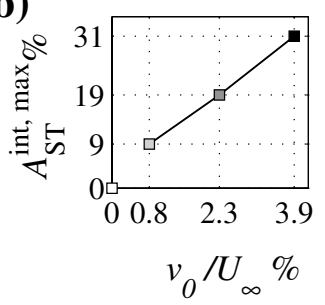

Fig. 7 a Streamwise evolution of the streak amplitude $A_{\mathrm{ST}}^{\text {int }}$ for three suction rates $v_{0} / U_{\infty}=0.8,2.3$ and 3.9\%.Darker symbols correspond to higher suction rate. The vertical dashed-dotted line displays the location of the downstream edge of the suction holes in the array, i.e., $x_{\mathrm{S}}=242 \mathrm{~mm}$. The inset shows a magnified display of the streak amplitude evolution for $v_{0} / U_{\infty}=2.3 \%$ with two extra points measured upstream of the suction array. The dashed-dotted lines display the upstream and downstream edges of the suction holes. b Maximum streak amplitude $A_{\mathrm{ST}}^{\text {int,max }}$ versus the suction rate 
amplifications in the streaky boundary layer, an integral measure has to be applied to the local TS wave amplitude in the entire cross-sectional plane for one spanwise wavelength of the suction holes in the array, $\Lambda$. Similar to previous investigations (e.g., Fransson et al. 2005a; Shahinfar et al. 2013; Sattarzadeh and Fransson 2015), this integral measure is here defined as

$A_{\mathrm{TS}}^{y z}(x)=\int_{-1 / 2}^{+1 / 2} \int_{0}^{\eta^{*}} \frac{A_{\mathrm{TS}}(x, \eta, \zeta)}{U_{\infty}} \mathrm{d} \eta \mathrm{d} \zeta$,

where $\eta^{*}=9$, as described for Eq. (1). The TS waves have a uniform spanwise distribution in the $2 \mathrm{D}$ reference base flow, and hence, wall-normal integration over one profile is analogous to the integral measure in the cross-sectional plane.

The TS wave amplification curves for the $\mathrm{C} 1$ configuration, where the TS waves are generated upstream of the suction plug, are shown in Fig. 8a and b for $F=100$ and $F=140$, respectively, and three suction rates $v_{0} / U_{\infty}=0.8$, 2.3 and $3.9 \%$. The open symbols display the amplification curves for the reference Blasius boundary layer and the filled symbols display the streaky boundary layer cases. The darker symbols correspond to higher suction rates. It is shown that the amplification of the TS waves is attenuated in the streaky boundary layer for all the cases, since a lower TS wave amplitude is obtained at branch II compared to the reference Blasius boundary layer. Prior to this attenuation, however, an initial amplification response of the TS waves with $F=140$ is observed for the two highest suction rates. The initial response is similar to the one observed for the miniature vortex generators (Shahinfar et al. 2013; Sattarzadeh and Fransson 2015), which as reported by Sattarzadeh and Fransson (2014) and Siconolfi et al. (2015b) results from the unstable modes generated in the low-speed region formed in the wake of the physical devices. Noteworthy is that the initial response is present even in the absence of any wake region of the physical devices.

The cross-sectional contour plots of the local TS wave amplitude $A_{\mathrm{TS}}$ at the downstream location of the maximum initial response for $\mathrm{C} 1$ configuration and $F=100$ is shown in Fig. 9. For the lowest suction rate $v_{0} / U_{\infty}=0.8 \%$, with no initial response displayed in the integral measure $A_{\mathrm{TS}}^{y z}$ (see Fig. 8a), local TS wave amplification is observed at $\zeta=-1 / 2$ and $1 / 2$, i.e., in the low-speed regions. Here, this amplification is nullified with the attenuated TS wave amplitude around the centre of the spanwise domain. However, for higher suction rates, where the initial response is observed, the TS waves are also amplified around the centre. This is corresponding to the formation of higher wallnormal gradients in the mean flow at the high-speed region (Sattarzadeh and Fransson 2014).

For both TS wave reference cases displayed in Fig. 8, the stable region following the initial response is extended by increasing the suction rate as branch I in the streaky boundary layer is moved further downstream (see the vertical dashed-dotted lines in the figure). On the other hand, the downstream location of branch II is not altered comparably in the presence of the streamwise streaks. Note that for the highest suction rate at $F=140$ the unstable region between branch I and II of the reference case is entirely stabilized.

The TS wave amplification curves for the $\mathrm{C} 2$ configuration, where the TS waves are generated downstream of the suction plug, are shown in Fig. 10a and b for $F=100$ and $F=140$, respectively, and the suction rate $v_{0} / U_{\infty}=2.3 \%$. For this configuration, no initial amplification of the TS waves is observed in the streaky boundary layer, which is similar to the results reported by Fransson et al. (a)

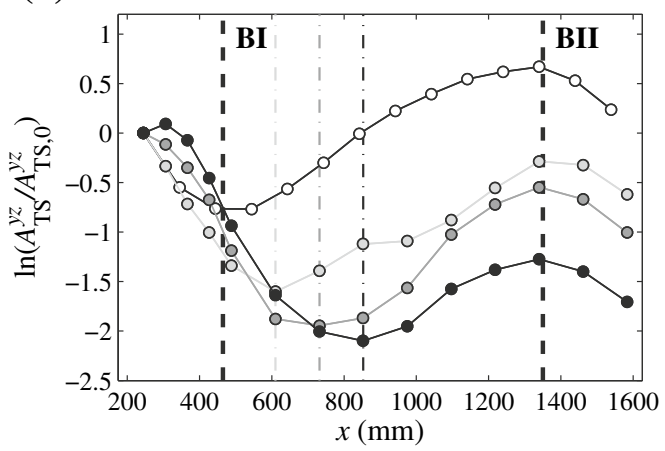

(b) Suction downstream of TSW ( $F=140)$

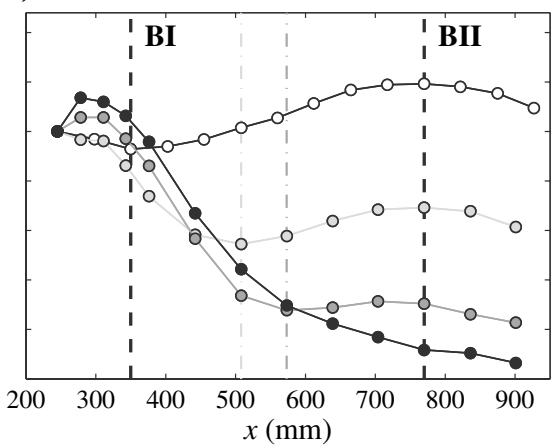

Fig. 8 Effect of suction on the TS wave amplification curve for three suction rates $v_{0} / U_{\infty}=0.8,2.3$ and $3.9 \%$. The suction is applied downstream of the TS wave generation location. The amplification curve for the reference Blasius case is shown with open symbols and the filled symbols display the streaky boundary layer cases. The darker symbols correspond to higher suction rate. $\mathbf{a}$ and $\mathbf{b}$ correspond to $F=100$ and $F=140$, respectively. The vertical dashed lines display the branch I and branch II of the neutral stability curve for the reference case. The vertical dashed-dotted lines display the altered location of branch I in the streaky boundary layers with colors corresponding to different suction rates 


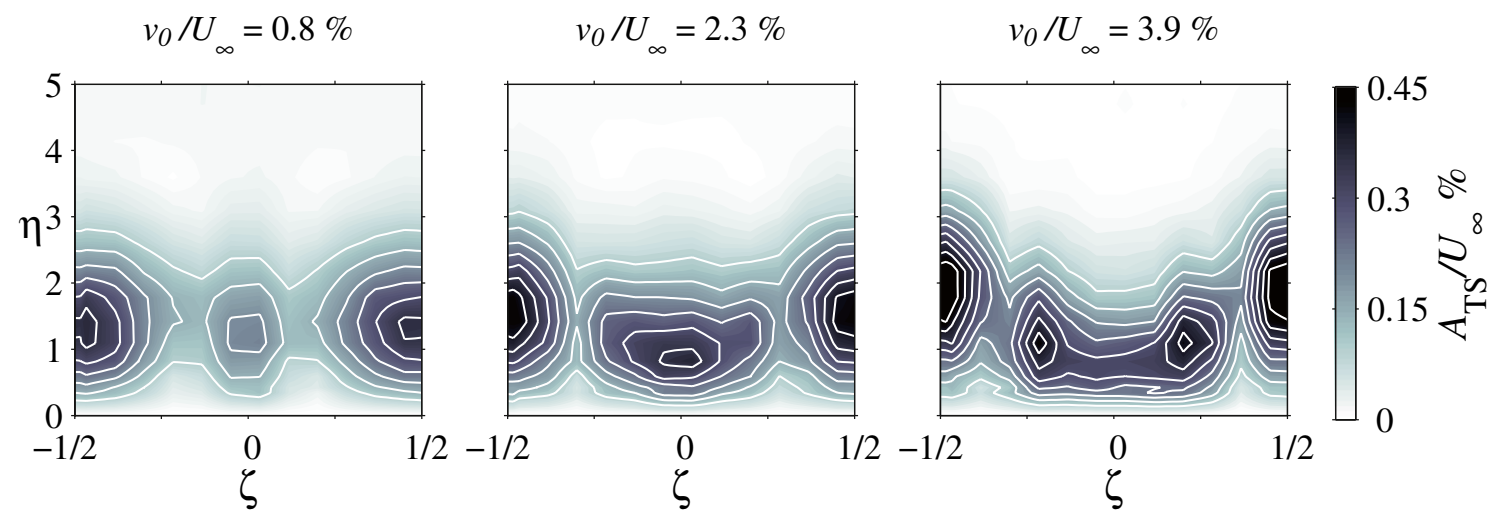

Fig. 9 Cross-sectional contour plots of the local TS wave amplitude $A_{\mathrm{TS}}$ for three suction rates $v_{0} / U_{\infty}=0.8,2.3$ and $3.9 \%$ from left to right, respectively, and at the downstream location of the maximum initial response, i.e., $x=306 \mathrm{~mm}$. All values are normalized with $U_{\infty}$. The suction is applied downstream of the TS wave generation location and $F=100$

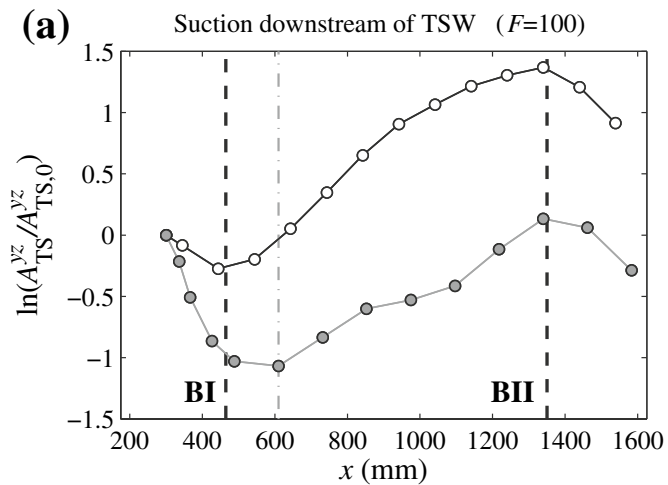

(b) Suction downstream of TSW $\quad(F=140)$

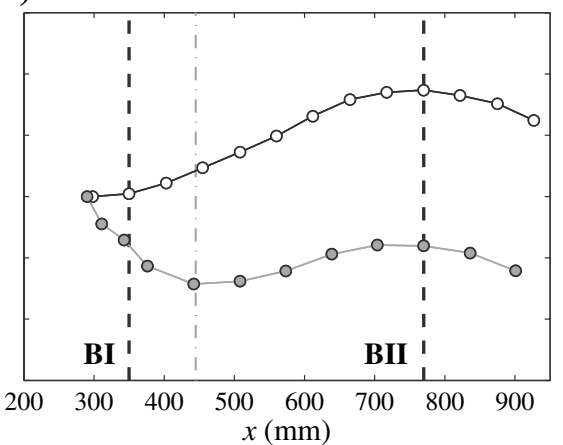

Fig. 10 The effect of suction on the TS wave amplification curve for $v_{0} / U_{\infty}=2.3 \%$. The suction is applied upstream of the TS wave generation location. The amplification curve for the reference Blasius case is shown with open symbols and the filled symbols display the streaky boundary layer cases. $\mathbf{a}$ and $\mathbf{b}$ correspond to $F=100$ and

(2005a), where the TS waves were generated downstream of a spanwise array of circular roughness elements. Similar to $\mathrm{C} 1$ configuration, branch I is moved downstream in the streaky boundary layer, and the TS amplitude is attenuated at branch II location compared to the reference Blasius boundary layer.

\section{Transition delay}

To test the laminar flow control the SVG method has to be applied to non-linear disturbances that force the boundary layer to breakdown to turbulence when the control is off. In this section, the stabilizing effect of the streaky boundary layer generated by discrete suction is investigated, where high-amplitude non-linear disturbances are generated in the boundary layer. The disturbances that are studied here consist of: (i) artificially forced single-frequency TS waves
$F=140$, respectively. The vertical dashed lines display the branch I and branch II of the neutral stability curve for the reference case. The vertical dashed-dotted lines display the altered location of branch I in the streaky boundary layers

generated by means of blowing and suction through the disturbance slots in the flat plate and (ii) natural TS waves that are manifested as a broad frequency band in the boundary layer at high free-stream velocity $U_{\infty}=14.5 \mathrm{~m} \mathrm{~s}^{-1}$.

\subsection{Artificially forced non-linear Tollmien-Schlichting waves}

For high initial forcing amplitudes of single-frequency TS waves, the harmonics of the forcing frequency are excited through non-linear effects. The fundamental forcing frequency and its harmonics undergo exponential growth in the region between branch I and branch II of neutral stability curve. Consequently, the triggered non-linearities in the shear flow force the boundary layer to undergo a sharp secondary instability and breakdown to turbulence downstream of branch II. However, for considerably high initial 


\section{$x(\mathrm{~mm})$}

(a)

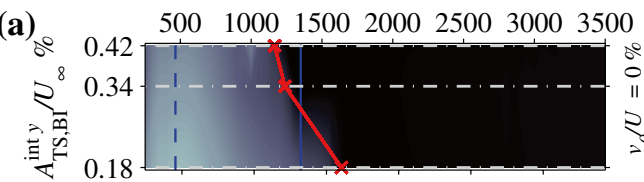

(b)

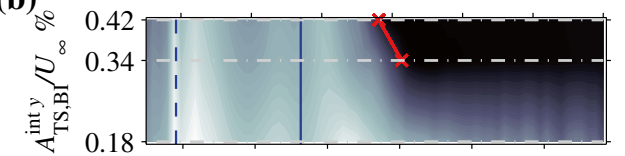

(c)

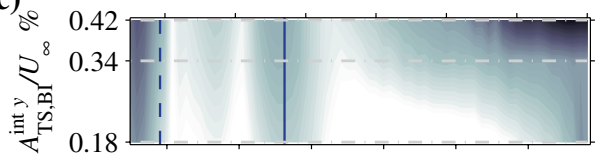

(d)

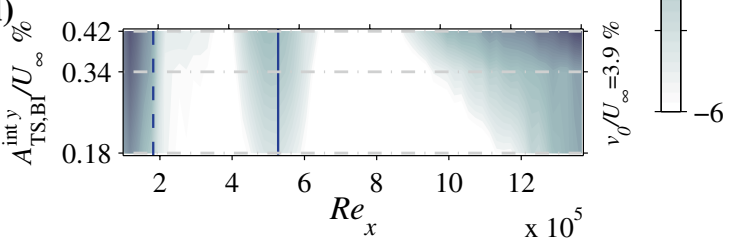

Fig. 11 Streamwise evolution of the disturbance energy versus TS waves amplitude at branch I (indicated by horizontal dashed-dotted lines) measured at $y / \delta_{1}=0.5$ and $\zeta=0$. The suction is applied downstream of the TS wave generation location and $F=100$. a Corresponds to the reference Blasius boundary layer, and $\mathbf{b}, \mathbf{c}$ and $\mathbf{d}$ correspond to suction rates $v_{0} / U_{\infty}=0.8,2.3$ and $3.9 \%$, respectively. Vertical dashed and solid lines correspond to the branch I and branch II locations of the reference case. Transition locations corresponding to $\gamma=0.5$ are plotted with $(\times)$-symbols

forcing amplitudes, the transition can even occur prior to the location of branch II.

To examine the robustness of the control mechanism, three levels of high initial forcing amplitude were investigated. The streamwise evolution of the disturbance energy in the reference Blasius boundary layer is shown in Fig. 11a. Here, the wall-normal integral of the normalized TS wave amplitude at branch I location $A_{\mathrm{TS} . \mathrm{BI}}^{\mathrm{int}} / U_{\infty}$ is displayed on the vertical axis as a measure of the initial forcing amplitude, and the measured cases are shown by horizontal dashed-dotted lines. The transition locations corresponding to the intermittency value of $\gamma=0.5$ are plotted with $(\mathrm{X})$-symbols. The same procedure as outlined in Fransson et al. (2005b) was used here to calculate $\gamma$. The intermittency is a statistical measure of the proportion of laminar versus turbulent flow sweeping the hot-wire probe. Branches I and II of the neutral stability curve for the reference Blasius boundary layer are also plotted as vertical dashed and solid lines, respectively. It is shown that for the lowest initial forcing amplitude, the transition occurs downstream of branch II, whereas by increasing the forcing amplitude, it is moved upstream and for the higher initial forcing amplitudes transition even takes place upstream of branch II.

The disturbance energy evolution in the streakyboundary layer for $\mathrm{C} 1$ configuration and suction rates $v_{0} / U_{\infty}=0.8,2.3$ and $3.9 \%$ is displayed in Fig. 11a-c, respectively. It is observed that in all the measured cases, the control has successfully delayed the transition to turbulence. For the lowest suction rate $v_{0} / U_{\infty}=0.8 \%$ (see Fig. 11b), transition still occurs within the downstream measured region, but it has moved further downstream compared to the uncontrolled cases for the two highest initial forcing amplitudes. Noteworthy is that the streaky boundary layer does not promote the transition to turbulence in any of the investigated cases. For the lowest forced TS wave amplitude, discrete suction of $v_{0} / U_{\infty}=0.8 \%$ leads to a delay in transition location of a least $120 \%$. The transition delay measurements were also successfully tested for the $\mathrm{C} 2$ configuration at the suction rate $v_{0} / U_{\infty}=2.3 \%$ (not shown here).

\subsection{Natural transition}

In the natural transition scenario, TS waves in a broad frequency band are produced in the boundary layer which simultaneously undergo exponential energy growth in the unstable region between the corresponding branch I and branch II on the neutral stability curve. The energy growth depends on the extent of the unstable region, which is a function of the frequency of the TS waves. For lower frequencies, a higher growth rate, i.e., $N$ factor, is obtained. This concept, or the so-called $e^{N}$ method (van Ingen 2008), is considered the basis for transition location estimations in boundary layer flows with low background disturbance level. Depending on the boundary layer parameters, the shear layer withstands a certain maximum growth rate prior to its breakdown to turbulence. The branch II location of the corresponding frequency range for this maximum growth rate would then predict the transition location. Now, it can be inducted that by decreasing the growth rate for the excited frequency band in the boundary layer, the maximum growth rate would correspond to a lower frequency range with a further downstream branch II location in the neutral stability curve. This would consequently lead to a delay in the transition location of the boundary layer.

Figure 12a displays the streamwise evolution of the disturbance energy in the boundary layer for $U_{\infty}=14.5 \mathrm{~m} \mathrm{~s}^{-1}$ for the uncontrolled and controlled cases with the suction rates $v_{0} / U_{\infty}=0$ and $0.9 \%$, respectively. The suction rate $v_{0} / U_{\infty}=0.9 \%$ generates a streaky boundary layer with $A_{\mathrm{ST}}^{\mathrm{int}, \mathrm{max}}=19 \%$. It is observed that the transition location corresponding to $\gamma=0.5$ is delayed by $30 \%$, i.e., from $R e_{x}=1.5 \times 10^{6}$ to $R e_{x}=1.95 \times 10^{6}$ in the controlled case. The corresponding normalized energy spectra 
Fig. 12 a Disturbance energy evolution for $U_{\infty}=14.5 \mathrm{~m} \mathrm{~s}^{-1}$ and measured at $y / \delta_{1}=0.5$ and $\zeta=0$. (Open circle)- and (open square)-symbols correspond to the suction rates $v_{0} / U_{\infty}=0$ and $0.9 \%$, respectively. The colorbar applied to the symbols correspond to the intermittency $(\gamma)$ of the velocity signals. Vertical dashed and dashed-dotted lines display the transition location corresponding to intermittency $\gamma=0.5$ for the uncontrolled and controlled cases, respectively. b and $\mathbf{c}$ show the corresponding normalized energy spectra $\left(e_{f}^{*}\right)$ evolution for the suction rates $v_{0} / U_{\infty}=0$ and $0.9 \%$, respectively. The gray solid line displays the neutral stability curve for the reference boundary layer

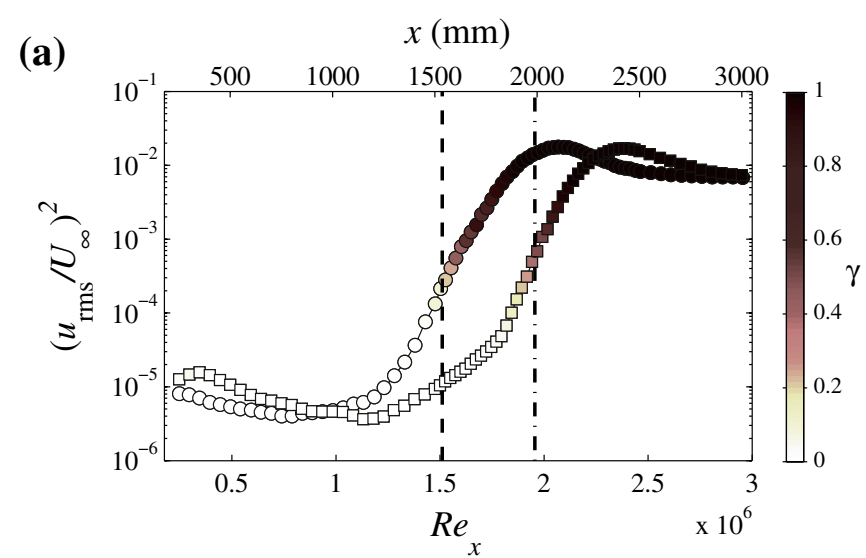

(b)

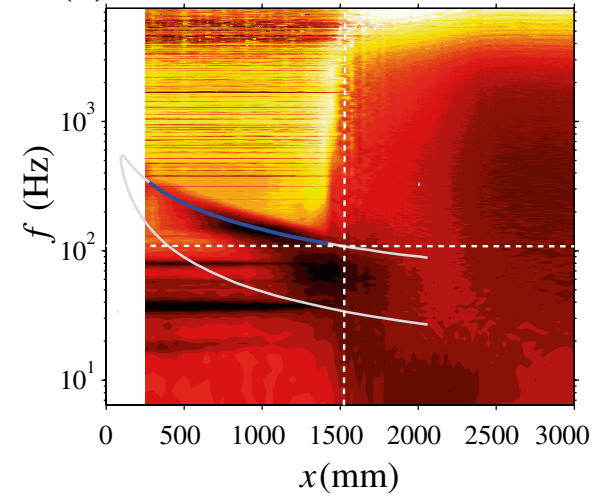

(c)

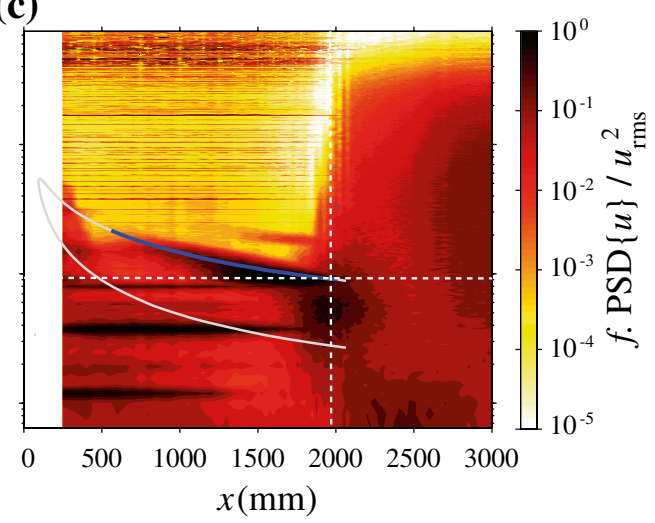

for the uncontrolled and controlled cases are displayed in Fig. $12 b$ and $c$, respectively. Here, the unscaled neutral stability curve for the reference Blasius boundary layer is also displayed with the gray solid line in both (b) and (c). The corresponding transition location for each case as shown in (a) is marked by the vertical dashed lines in (b) and (c). In the energy spectra for the uncontrolled case (Fig. 12b), two dominant modes with $f \approx 38$ and $80 \mathrm{~Hz}$ are excited in the boundary layer along with a broad frequency band in the range $110 \mathrm{~Hz} \leq f \leq 460 \mathrm{~Hz}$. It can be observed that disturbances in this frequency range become unstable upstream of their corresponding branch II location shown with blue color. For higher frequencies than $f \approx 110 \mathrm{~Hz}$ (marked by the horizontal dashed line), the disturbance energy decays downstream of the branch II location as the boundary layer withstands the corresponding growth rate. However, at $f \approx 110 \mathrm{~Hz}$ the growth rate has reached its maximum and the boundary layer breaks down to turbulence. Note that $f \approx 110 \mathrm{~Hz}$ corresponds to the frequency, where the vertical dashed line (i.e., transition location) intercepts the branch II of the neutral stability curve. In the energy spectra for the controlled case (Fig. 12c), the same frequency band is excited in the beginning of the measurement domain; however, the disturbances with the frequency higher than $f \approx 210 \mathrm{~Hz}$ decay rapidly and do not grow in energy upstream to their branch II location. For this case, the vertical dashed line (i.e., transition location) intercepts the branch II of the neutral stability curve at a further downstream position, compared to the uncontrolled case, which corresponds to $f \approx 90 \mathrm{~Hz}$ (shown by the horizontal dashed line). This implies that the disturbance waves with $f>90 \mathrm{~Hz}$ (including $f \approx 110 \mathrm{~Hz}$ ) are stabilized in the streaky boundary layer and become stable after their corresponding branch II locations. As a result, the transition to turbulence is delayed by moving downstream of branch II for $f \approx 90 \mathrm{~Hz}$. Noteworthy is that in the streamwise domain, where the transition is delayed, the first harmonic of the attenuated frequency range is excited. The dominant modes with $f \approx 38$ and $80 \mathrm{~Hz}$ are also excited in the controlled case together with a lower frequency mode at $f \approx 13 \mathrm{~Hz}$ which, however, is damped prior to the transition location. This low-frequency mode is likely to be associated with the wind-tunnel facility and has negligible effect on the boundary layer transition scenario.

\section{Conclusions}

In the present investigation, the streaky boundary layers, generated by finite suction through discrete holes aligned 
in a spanwise-oriented array on a flat plate, have been studied. Steady and robust high-amplitude streaks of high- and low-speed regions were generated in the flat plate boundary layer through this technique which can be utilized in the spanwise mean velocity gradient method for laminar flow control. The spanwise modulations in the mean flow increases with suction rate due to higher induced circulation in the cross-sectional plane. The induced circulation also modulates the boundary layer upstream of the suction array. In the streaky boundary layer, the fundamental steady spanwise mode $\lambda_{1}$, corresponding to the spanwise wavelength of the suction holes in the array, has the highest amplitude and is the dominant mode as the boundary layer evolves downstream.

To test the laminar flow control technique, two measurement configurations were investigated, where artificially forced TS waves were generated upstream and downstream of the suction array. Despite the fact that no physical devices, i.e., roughness elements or miniature vortex generators, were utilized to generate the streaks, an initial growth of the TS waves was observed for the configuration, where the TS waves were generated upstream of the suction array. The initial growth increases for streaky boundary layers with higher maximum streak amplitudes, which is similar to the results reported for the miniature vortex generators. This initial growth was eliminated when the TS waves were generated downstream of the suction array.

In spite of the initial growth, the TS wave amplification is attenuated in the presence of the streaks which leads to a delay of transition to turbulence for high-amplitude nonlinear TS waves. Noteworthy is that the streaky boundary layer generated by discrete suction does not promote the transition to turbulence in any of the investigated cases. The laminar flow control was also tested successfully for natural transition scenario at high free-stream velocity. It was shown that the growth rate of the broad frequency band of TS waves generated in the uncontrolled case is attenuated in the streaky boundary layer case which leads to transition to turbulence delay.

So far, we have not made any effort in really quantifying how much suction we have used in our LFC technique, using the SVG method, with respect to any other known successful suction-control method. Hence, as a final remark, we feel obliged to relate the present volume-suction coefficient to the ASBL one from the introduction, which was obtained using the critical Reynolds number, namely $C_{q}^{*}=9.26 \times 10^{-8}$. This theoretical volume-suction coefficient does not allow any modal disturbance growth, and this, for a minimum skin-friction coefficient $\left(c_{\mathrm{f}}\right)$. Now, if we want to calculate a corresponding volume-suction coefficient for the present discrete suction case, we need the open over total area ratio. Here, we calculate the open area by adding the present 16 discrete suction hole areas. The total area, $(\Lambda \times 16) \times L$, is obtained by considering the spanwise distance of the 16 holes times a laminar downstream extent $(L)$ of at least $3500 \mathrm{~mm}$. With a suction rate of $2.3 \%$, one obtains $C_{q}^{*}=\left(2.30 \times 10^{-2}\right) \cdot\left(2.38 \times 10^{-4}\right)=5.48 \times 10^{-6}$. At this point, the volume-suction coefficient is two orders of magnitude larger than what would be required to keep the flow laminar applying uniform suction and does not sound very attractive. However, one has to point out that the ASBL $C_{q}^{*}$ value is nothing else than a theoretical limitation, and is unrealistic for most real experiments or flow applications (which is hardly ever mentioned). The entry length to reach the ASBL state can easily be estimated to over $29,000 \mathrm{~m}$ for a free-stream velocity of $6 \mathrm{~m} \mathrm{~s}^{-1}$ (as used here) (see Fig. 3 in Fransson and Alfredsson 2003). Looking at realistic values that would be applicable on, for instance, a commercial airliner, such as $U_{\infty}=210 \mathrm{~m} \mathrm{~s}^{-1}$ and $R e=750$, the entry length would be reduced to $0.16 \mathrm{~m}$. On the other hand, the suction velocity would be $0.28 \mathrm{~m} \mathrm{~s}^{-1}$ and the $c_{\mathrm{f}}$ value would increase by a factor of 72 compared to the critical condition of the ASBL. The volume-suction coefficient then becomes $C_{q}^{*}=6.67 \times 10^{-6}$ and hence is comparable with the present volume-suction coefficient. On the other hand, after the entry length of $0.16 \mathrm{~m}$ with a uniform suction velocity of $0.28 \mathrm{~m} \mathrm{~s}^{-1}$ and at the speed of a commercial airliner, $c_{\mathrm{f}}$ stays just below the expected value for a turbulent boundary layer, i.e., an order of magnitude larger compared to the stabilized boundary layer using the SVG method. Given this picture, we can draw the following conclusion about the present volume-suction coefficient; Applying suction through discrete holes to make use of the SVG method is probably a far better control approach than any other parameter choice using uniform suction, since the skin-friction coefficient is typically an order of magnitude smaller. Finally, as often pointed out, another advantage of relatively large discrete suction holes is that they are more easily cleaned as compared to porous materials or laser drilled surfaces, which may encounter problems with clogging in real flow applications.

Acknowledgements J. H. M. F. acknowledges the European Research Council (ERC) for its financial support of the AFRODITE project through a Starting Grant (StG): ERC-StG-2010-258339.

Open Access This article is distributed under the terms of the Creative Commons Attribution 4.0 International License (http:// creativecommons.org/licenses/by/4.0/), which permits unrestricted use, distribution, and reproduction in any medium, provided you give appropriate credit to the original author(s) and the source, provide a link to the Creative Commons license, and indicate if changes were made. 


\section{References}

Andersson P, Berggren M, Henningson DS (1999) Optimal disturbances and bypass transition in boundary layers. Phys Fluids 11:134-150

Bakchinov AA, Grek GR, Klingmann BGB, Kozlov VV (1995) Transition experiments in a boundary layer with embedded streamwise vortices. Phys Fluids 7:820-832

Boiko AV, Westin KJA, Klingmann BGB, Kozlov VV, Alfredsson PH (1994) Experiments in a boundary layer subjected to free stream turbulence. Part 2. The role of TS-waves in the transition process. J Fluid Mech 281:219-245

Cossu C, Brandt L (2002) Stabilization of Tollmien-Schlichting waves by finite amplitude optimal streaks in the Blasius boundary layer. Phys Fluids 14:L57-L60

Cossu C, Brandt L (2004) On Tollmien-Schlichting-like waves in streaky boundary layers. Eur J Mech/B Fluids 23:815-833

Downs RS, Fransson JHM (2014) Tollmien-Schlichting wave growth over spanwise-periodic surface patterns. J Fluid Mech 754:39-74

Drazin P, Reid W (1981) Hydrodynamic stability. Cambridge University Press, Cambridge

Elofsson PA, Kawakami M, Alfredsson PH (1999) Experiments on the stability of streamwise streaks in plane Poiseuille flow. Phys Fluids 11:915-930

Fransson JHM, Alfredsson PH (2003) On the disturbance growth in an asymptotic suction boundary layer. J Fluid Mech 482:51-90

Fransson JHM, Talamelli A (2012) On the generation of steady streamwise streaks in flat-plate boundary layers. J Fluid Mech 698:211-234

Fransson JHM, Brandt L, Talamelli A, Cossu C (2004) Experimental and theoretical investigation of the nonmodal growth of steady streaks in a flat plate boundary layer. Phys Fluids 16(10):3627-3638

Fransson JHM, Brandt L, Talamelli A, Cossu C (2005a) Experimental study of the stabilisation of Tollmien-Schlichting waves by finite amplitude streaks. Phys Fluids 17(054):110

Fransson JHM, Matsubara M, Alfredsson PH (2005b) Transition induced by free stream turbulence. J Fluid Mech 527:1-25

Fransson JHM, Talamelli A, Brandt L, Cossu C (2006) Delaying transition to turbulence by a passive mechanism. Phys Rev Lett 96(064):501

Gad-el-Hak M (2000) Flow control. Cambridge University Press, Cambridge

Gaponenko V, Kachanov Y (1994) New methods of generation of controlled spectrum instability waves in boundary layers. In: ICMAR, Part 1. Institute of Theoretical and Applied Mechanics, Novosibirsk, pp 125-130

Green JE (2008) Laminar flow control-back to the future? AIAA Paper, pp 2008-3738

Gregory N (1961) Research on suction surfaces for laminar flow. Bound Layer Flow Control II:924-957

Johansson AV, Alfredsson PH (1982) On the structure of turbulent channel flow. J Fluid Mech 122:295-314

Joslin RD (1998) Aircraft laminar flow control. Annu Rev Fluid Mech 30:1-29

Kachanov YS, Tararykin OI (1987) Experimental investigation of a relaxating boundary layer. Izv Sib Otd Akad Nauk SSSR Ser Tech Nauk 18:9

Landahl MT (1980) A note on an algebraic instability of inviscid parallel shear flows. J Fluid Mech 98:243-251

Lindgren B, Johansson AV (2002) Evaluation of the flow quality in the MTL wind-tunnel. KTH Mechanics report KTH/MEK/ TR02/13SE
Luchini P (1996) Reynolds-number independent instability of the boundary layer over a flat surface. J Fluid Mech 327:101-115

Luchini P (2000) Reynolds-number independent instability of the boundary layer over a flat surface. Part 2: Optimal perturbations. J Fluid Mech 404:289-309

MacManus DG, Eaton JA (2000) Flow physics of discrete boundary layer suction-measurements and predictions. J Fluid Mech 417:47-75

Pfenninger W, Groth E (1961) Low drag boundary layer suction experiments in flight on a wing glove of an F-94A airplane with suction through a large number of fine slots. Bound Layer Flow Control II:981-999

Reynolds GA, Saric WS (1986) Experiments on the stability of the flat-plate boundary layer with suction. AIAA J 24:202-207

Roberts PJD, Floryan JM, Casalis G, Arnal D (2001) Boundary layer instability induced by surface suction. Phys Fluids 13:2543-2552

Sattarzadeh SS, Fransson JHM (2014) Experimental investigation on the steady and unsteady disturbances in a flat plate boundary layer. Phys Fluids 26(12):124103

Sattarzadeh SS, Fransson JHM (2015) On the scaling of streamwise streaks and their efficiency to attenuate Tollmien-Schlichting waves. Exp Fluids 56(3):1-16

Sattarzadeh SS, Fransson JHM (2016) Mastering non-linear flow dynamics for laminar flow control. Phys Rev E 94:021103(R)

Sattarzadeh SS, Fransson JHM, Talamelli A, Fallenius BEG (2014) Consecutive turbulence transition delay with reinforced passive control. Phys Rev E 89(061):001(R)

Schlatter P, Deusebio E, de Lange R, Brandt L (2010) Numerical study of the stabilisation of boundary-layer disturbances by finite amplitude streaks. Int J Flow Control 2:259-288

Schlichting H (1933) Berechnung der anfachung kleiner störungen bei der plattenströmung. Z Angew Math Mech 13:171-174

Schlichting H (1979) Boundary layer theory. McGraw-Hill, New York

Schubauer GB, Skramstad HK (1947) Laminar boundary layer oscillations and the stability of laminar flow. J Aero Sci 14:69-78

Shahinfar S, Sattarzadeh SS, Fransson JHM, Talamelli A (2012) Revival of classical vortex generators now for transition delay. Phys Rev Lett 109(074):501

Shahinfar S, Fransson JHM, Sattarzadeh SS, Talamelli A (2013) Scaling of streamwise boundary layer streaks and their ability to reduce skin-friction drag. J Fluid Mech 733:1-32

Shahinfar S, Sattarzadeh SS, Fransson JHM (2014) Passive boundary layer control of oblique disturbances by finite amplitude streaks. J Fluid Mech 749:1-36

Siconolfi L, Camarri S, Fransson JHM (2015a) Boundary layer stabilization using free-stream vortices. J Fluid Mech 764:R2

Siconolfi L, Camarri S, Fransson JHM (2015b) Stability analysis of boundary layers controlled by miniature vortex generators. J Fluid Mech 784:596-618

Squire HB (1933) On the stability for three-dimensional disturbances of viscous fluid flows between parallel walls. Proc R Soc Lond A 142(847):621-628

Tani I, Komoda H (1962) Boundary layer transition in the presence of streamwise vortices. J Aerosp Sci 29:440

Tollmien W (1929) Über die entstehung der turbulenz. Nachr Ges Wiss Göttingen 21-24 [English translation NACA TM 609 (1931)]

Trip R, Fransson JHM (2014) Boundary layer modification by means of wall suction and the effect on the wake behind a rectangular forebody. Phys Fluids 26(125):105

van Ingen JL (2008) The $\mathrm{e}^{\mathrm{n}}$ method for transition prediction. Historical review of work at tu delft. AIAA Paper, pp 2008-3830 
Westin KJA, Boiko AV, Klingmann BGB, Kozlov VV, Alfredsson PH (1994) Experiments in a boundary layer subject to free-stream turbulence. Part i: Boundary layer structure and receptivity. J Fluid Mech 281:193-218

White EB (2002) Transient growth of stationary disturbances in a flat plate boundary layer. Phys Fluids 14:4429-4439
White EB, Rice JM, Ergin FG (2005) Receptivity of stationary transient disturbances to surface roughness. Phys Fluids 17(064):109 Yoshioka S, Fransson JHM, Alfredsson PH (2004) Free stream turbulence induced disturbances in boundary layers with wall suction. Phys Fluids 16:3530-3539 